\title{
On eigenfunction expansion of solutions to the Hamilton equations
}

\author{
A. Komech1 1 \\ Faculty of Mathematics of Vienna University \\ and Institute for Information Transmission Problems RAS \\ e-mail: alexander.komech@univie.ac.at \\ E. Kopylova目 \\ Faculty of Mathematics of Vienna University \\ and Institute for Information Transmission Problems RAS \\ e-mail: elena.kopylova@univie.ac.at
}

\begin{abstract}
We establish a spectral representation for solutions to linear Hamilton equations with positive definite energy in a Hilbert space. Our approach is a special version of M. Krein's spectral theory of $J$-selfadjoint operators in the Hilbert spaces with indefinite metric. Our main result is an application to the eigenfunction expansion for the linearized relativistic Ginzburg-Landau equation.
\end{abstract}

Key words and phrases: Hamilton equation; selfadjoint operator; $J$-selfadjoint operator; Krein space; spectral resolution; spectral representation; Ginzburg-Landau equation; kink; asymptotic stability; eigenvector; generalized eigenfunction; eigenfunction expansion; Fermi Golden Rule.

\footnotetext{
${ }^{1}$ Supported partly by Alexander von Humboldt Research Award, Austrian Science Fund (FWF): P22198-N13, and the grant of the Russian Foundation for Basic Research.

${ }^{2}$ Supported partly by Austrian Science Fund (FWF): M1329-N13, and the grant of the Russian Foundation for Basic Research.
} 


\section{Introduction}

We consider complex linear Hamilton operators in a complex Hilbert space $\mathscr{X}$,

$$
A=J B, \quad \text { where } \quad B^{*}=B, \quad J^{*}=-J, J^{2}=-1 .
$$

In other words, $A$ is a $J$-selfadjoint operator [35]. The selfadjoint operator $B$ is defined on a dense domain $D(B) \subset \mathscr{X}$. Our aim is a spectral representation for solutions to the equation

$$
\dot{X}(t)=A X(t), \quad t \in \mathbb{R} .
$$

Our main goal is an application to the eigenfunction expansion for the linearized relativistic GinzburgLandau equation.

In the simplest case, when $J=i$, the solutions are given by $X(t)=e^{i B t} X(0)$. A more general 'commutative case', when $J B=B J$, reduces to the case $J=i$ since $J B=i B_{1}$, where $B_{1}=-i J B$ is the selfadjoint operator. However, $J B \neq B J$ for linearizations of $U(1)$-invariant nonlinear Schrödinger equations as we show in Appendix B.

The complex Hamilton operators arise as the complexification of a linearization of nonlinear Hamilton equations in a real Hilbert space $\mathscr{X}_{r}$,

$$
\dot{\psi}_{r}(t)=J_{r} D \mathscr{H}\left(\psi_{r}(t)\right)
$$

where $\mathscr{H}$ is the Hamilton functional, $J_{r}^{*}=-J_{r}, J_{r}^{2}=-1$, and $D \mathscr{H}$ stands for the differential which is a linear operator over real numbers. The linearization of (1.3) at a stationary state $S \in \mathscr{X}_{r}$ reads as

$$
\dot{X}_{r}(t)=A_{r} X_{r}(t), \quad A_{r}=J_{r} B_{r},
$$

where $B_{r}:=D D \mathscr{H}(S)$ is a real symmetric operator. This is the Hamilton system with the Hamilton functional

$$
\mathscr{H}_{0}\left(X_{r}\right)=\frac{1}{2}\left\langle B_{r} X_{r}, X_{r}\right\rangle_{r},
$$

where the brackets denote the scalar product on $\mathscr{X}_{r}$. The complexification of the operator $A_{r}$ in the complex space $\mathscr{X}=\mathscr{X}_{r} \oplus i \mathscr{X}_{r}$ reads as $(1.1)$, where $J, B$ are the complexifications of $J_{r}, B_{r}$ respectively. Moreover, $B$ is complex selfadjoint on $\mathscr{X}$ if $B_{r}$ is real selfadjoint on $\mathscr{X}_{r}$.

The representation of solutions to the Hamilton equations in the form of oscillatory integrals is indispensable in the proof of the dispersion decay for linearized equations in the theory of asymptotic stability of solitary waves of nonlinear Schrödinger, wave, Klein-Gordon, Maxwell, Dirac and relativistic Ginzburg-Landau equations, which were extensively developed the last two decades [3]-[7], [9]-[16], [28]-[31], [40]-[42], and [52]-[58]. However, many features of these representations were not justified up to now, since the generators of the linearized equations can be non symmetric, as noted in Introduction of [13]. In particular, the eigenfunction expansion, used in [30], was not justified with detail.

We fill this gap in the simplest case of positive definite 'energy operators' $B$ satisfying the following spectral condition:

Condition $\quad \mathbf{P}_{+} \quad \sigma(B) \subset[\delta, \infty), \quad \delta>0$. 
Equivalently, $B$ is invertible in $\mathscr{X}$ and $B>0$. This framework is sufficient for our main application to the linearized relativistic Ginzburg-Landau equation.

Our results are the following:

- The similarity of $i A$ to a selfadjoint operator.

- The existence and uniqueness of generalized solutions to (1.2) for all initial states $X$ with finite energy $\langle B X, X\rangle$, where $\langle\cdot, \cdot\rangle$ stands for the scalar product on $\mathscr{X}$.

- A spectral representation of solutions for complex and real Hamilton generators.

- Our main result is the eigenfunction expansion

$$
\left(\begin{array}{c}
\psi(t) \\
\dot{\psi}(t)
\end{array}\right)=\sum_{-N}^{N} e^{-i \omega_{k} t} C_{k} a_{k}+\int_{|\omega| \geq m} e^{-i \omega t} C(\omega) a_{\omega} d \omega
$$

for solutions to the linearized relativistic nonlinear Ginzburg-Landau equation [30]. Here $a_{k}$ are the eigenfunctions of the corresponding Klein-Gordon generator

$$
A=\left(\begin{array}{cc}
0 & 1 \\
-H_{0} & 0
\end{array}\right)
$$

where $H_{0}:=-\frac{d^{2}}{d x^{2}}+m^{2}+V_{0}(x)$. Finally, $a_{\omega}$ are the generalized eigenfunctions of the continuous spectrum of $A$.

Such eigenfunction expansions were used in [6, 7, 30] for the calculation of 'Fermi Golden Rule' (FGR) in the context of nonlinear Schrödinger and Klein-Gordon equations. This is a nondegeneracy condition introduced in [52] in the framework of nonlinear wave and Schrödinger equations. The condition provides a strong coupling of discrete and continuous spectral components of solutions, which provides the energy radiation to infinity and results in the asymptotic stability of solitary waves. The calculation of FGR, as given in [6, 7, 30], relies on eigenfunction expansions of type (1.7). Our main Theorem 5.4]justifies the eigenfunction expansion [30, (5.14)] which was not proved with detail. This justification was one of our main motivation in writing the present paper.

Let us comment on our approach. First, we reduce the problem to a selfadjoint generator defined uniquely by $A$ justifying the classical M. Krein transformation under condition (1.6). This reduction is a special version of spectral theory of $J$-selfadjoint operators in the Hilbert spaces with an indefinite metric [2, 33]. We plan to extend elsewhere these methods and results to more general degenerated case when $\operatorname{Ker} B \neq 0$.

Second, we apply the abstract spectral theory to the operator (1.8) and develop a modification of the eigenfunction expansion theory [45, pp 114-115] for the reduced selfadjoint operator, see Remark 4.1. Finally, we apply the theory of PDO to deduce (1.7) from this modification.

One of the novelties here is a vector-valued treatment of the convergence of the integral over the continuous spectrum in (1.7). Namely, we show that the integral is the limit of the corresponding integrals over $m \leq|\omega| \leq M$ as $M \rightarrow \infty$ in the Sobolev space $H^{1}(\mathbb{R}) \oplus L^{2}(\mathbb{R})$. In its own turn, the integral over $m \leq|\omega| \leq M$ is absolutely converging in the weighted $L^{2}$-space with the weight $(1+$ $|x|)^{-s}$ where $s>1$.

Let us comment on related works. Some spectral properties of the Hamilton non-selfadjoint operators were studied by V. Buslaev and G. Perelman [5, 6, 7], M.B. Erdogan and W. Schlag [17, 49], 
S. Cuccagna, D. Pelinovsky and V. Vougalter [11]. The eigenfunction expansions of $J$-selfadjoint operators were not justified previously.

The spectral resolution of bounded $J$-selfadjoint nonnegative operators in the Krein spaces was constructed by M. Krein, H. Langer and Yu. Smul'jan [33, 34], and extended to unbounded definitizable operators by M. Krein, P. Jonas, H. Langer and others [21, 22, 24, 35, 36]. The corresponding unitary operators were considered by P. Jonas [23]. However, the spectral resolution alone is insufficient for a justification of eigenfunction expansions. Our version of the theory under condition (1.6) allows us to justify the eigenfunction expansion (1.8).

The spectral theory of definitizable operators was applied to the Klein-Gordon equations with non-positive energy by P. Jonas, H. Langer, B. Najman and C. Tretter [25, 26, 37, 38, 39], where the existence and uniqueness of classical solutions were proved, and the existence of unstable eigenvalues (imaginary frequencies) was studied. The instability is related to the known Klein paradox in quantum mechanics [48].

The scattering theory for the Klein-Gordon equations with non-positive energy was developed by C. Gérard and T. Kako using the theory of the definitizable operators in the Krein spaces [18, 27].

The plan of our paper is as follows. In Section 2 we justify the M. Krein transformation under condition (1.6), and construct a unitary dynamical group and its spectral representation. In Section 3 we check condition (1.6) for the operator (1.8), and in Sections 4-6 we justify the eigenfunction expansion (1.7) applying the methods of Section 2. In Appendix A we check the spectral condition (1.6) for a class of operators, and in Appendix B we check that $J B \neq B J$ for linearizations of nonlinear Schrödinger equations.

Acknowledgments The authors thank V. Ivrii, A. Kostenko, M. Malamud and G. Teschl for useful discussions on pseudodifferential operators and $J$-selfadjoint operators.

\section{Spectral representation}

We are going to obtain a spectral representation for solutions to equation (1.2).

\subsection{Generalized solutions}

Let $D(B)$ denote the dense domain of the selfadjoint operator $B$. We set $\Lambda:=B^{1 / 2}>0$ and denote by $\mathscr{V} \subset \mathscr{X}$ the Hilbert space which is the domain of $\Lambda$ endowed with the norm

$$
\|X\|_{\mathscr{V}}:=\|\Lambda X\|_{\mathscr{X}}
$$

which is positive definite by (1.6). We have a continuous injection of Hilbert spaces $\mathscr{V} \subset \mathscr{X}$, and the unitary operator

$$
\Lambda: \mathscr{V} \rightarrow \mathscr{X}
$$

In particular,

$$
\Lambda^{-1}: \mathscr{X} \rightarrow \mathscr{V}
$$

is a bounded operator. For example, $\mathscr{V}$ is the Sobolev space $H^{1}\left(\mathbb{R}^{n}\right)$ in the case of $\mathscr{X}=L^{2}\left(\mathbb{R}^{n}\right)$ and operator $A=i\left(-\Delta+m^{2}\right)$ with any real $m \neq 0$.

Finally, $J$ is the unitary operator in $\mathscr{X}$ by $(1.1)$, and hence

$$
A^{-1}=-B^{-1} J: \mathscr{X} \rightarrow \mathscr{V}
$$


is a bounded operator by (2.3). We will consider solutions

$$
X(t) \in C(\mathbb{R}, \mathscr{V})
$$

to equation (1.2). We will understand the equation in the sense of mild solutions [8]

$$
X(t)-X(0)=A \int_{0}^{t} X(s) d s, \quad t \in \mathbb{R} .
$$

By (2.4) this is equivalent to the identity

$$
A^{-1}[X(t)-X(0)]=\int_{0}^{t} X(s) d s, \quad t \in \mathbb{R},
$$

where the Riemann integral converges in $\mathscr{V}$ by (2.5).

\subsection{Krein transformation}

We apply well known formal similarity transformation

$$
A=J \Lambda^{2} \rightarrow \Lambda A \Lambda^{-1}=\Lambda J \Lambda,
$$

rising to M. Krein. This transformation corresponds to the substitution

$$
Z(t):=\Lambda X(t) \in C(\mathbb{R}, \mathscr{X}),
$$

used by M. Krein in the theory of parametric resonance: see formula (1.40) of [19, Chapter VI]. Applying the transformation (2.8) to the both sides of (1.2), we obtain formally the corresponding 'Schrödinger equation'

$$
i \dot{Z}(t)=H Z(t), \quad t \in \mathbb{R} .
$$

Here $H$ stands for the 'Schrödinger operator'

$$
H=\Lambda i J \Lambda
$$

which is symmetric on the domain

$$
D(H)=\{X \in \mathscr{V}: J \Lambda X \in \mathscr{V}\} .
$$

These arguments give all solutions to (1.2) in the case of finite dimensional space $\mathscr{X}$. In the infinite dimensional case the problem is less trivial.

\subsection{Selfadjoint generator}

We must justify that the operator $H$ is densely defined and selfadjoint in our situation. Otherwise, the formal transformation (2.8) would not help to construct solutions of equation (1.2).

Lemma 2.1. Let condition (1.6) hold. Then $D(H)$ is dense in $\mathscr{X}$ and the operator $H$ is selfadjoint. 
Proof The operator $H$ is injective. Further, $\operatorname{Ran} \Lambda=\mathscr{X}$ by $(1.6)$, and $J: \mathscr{X} \rightarrow \mathscr{X}$ is the unitary operator. Hence, $\operatorname{Ran} H=\mathscr{X}$. Consider the inverse operator

$$
R:=H^{-1}=\Lambda^{-1} i J \Lambda^{-1}
$$

It is selfadjoint since $D(R)=\operatorname{Ran} H=\mathscr{X}$ and $R$ is bounded and symmetric. Finally, $R$ is injective, and hence,

$$
H=R^{-1}
$$

is a densely defined selfadjoint operator by Theorem 13.11 (b) of [47]:

$$
H^{*}=H, \quad D(H)=\operatorname{Ran} R \subset \mathscr{V} .
$$

Thus, the lemma is proved.

As a result, under condition (1.6) the identity

$$
A=-i \Lambda^{-1} H \Lambda
$$

holds on the domain $\Lambda^{-1} D(H)$, which is dense in $\mathscr{V}$. We will understand equation (2.10) similarly to (2.7):

$$
i H^{-1}[Z(t)-Z(0)]=\int_{0}^{t} Z(s) d s, \quad t \in \mathbb{R},
$$

where the Riemann integral converges in $\mathscr{X}$ by 2.9 .

Corollary 2.2. Let condition (1.6) hold. Then for any $Z(0) \in \mathscr{X}$ equation (2.10) admits a unique solution $Z(t) \in C(\mathbb{R}, \mathscr{X})$ in the sense (2.16). The solution is given by

$$
Z(t)=e^{-i H t} Z(0) \in C(\mathbb{R}, \mathscr{X})
$$

\subsection{Unitary dynamical group}

It is easy to check that equation (2.10) for $Z(t) \in C(\mathbb{R}, \mathscr{X})$ in the sense (2.16) is equivalent to equation (1.2) in the sense (2.7) for

$$
X(t)=\Lambda^{-1} Z(t) \in C(\mathbb{R}, \mathscr{V}) .
$$

Hence, Corollary 2.2 implies the following lemma.

Lemma 2.3. Let condition (1.6) hold. Then

i) For any $X(0) \in \mathscr{V}$, the function

$$
X(t)=\Lambda^{-1} e^{-i H t} \Lambda X(0) \in C(\mathbb{R}, \mathscr{V})
$$

is the unique solution to (1.2) in the sense (2.7).

ii) The dynamical group $V(t): X(0) \mapsto X(t)$ is unitary in $\mathscr{V}$, since

$$
\|X(t)\|_{\mathscr{V}}:=\|\Lambda X(t)\|_{\mathscr{X}}=\left\|e^{-i H t} \Lambda X(0)\right\|_{\mathscr{X}}=\|\Lambda X(0)\|_{\mathscr{X}}=:\|X(0)\|_{\mathscr{V}} .
$$




\subsection{Spectral resolution}

Let $E(\omega)$ denote the spectral resolution of the selfadjoint operator $H$; i.e.,

$$
H Z=\int \omega d E(\omega) Z, Z \in D(H)
$$

the Riemann-Stieltjes integrals converging in $\mathscr{X}$. This resolution and (2.19) imply the following proposition.

Proposition 2.4. Let condition (1.6) hold. Then the following spectral representation holds for solutions to the complex Hamilton equations (1.2):

$$
X(t)=\Lambda^{-1} \int e^{-i \omega t} d E(\omega) \Lambda X(0), X(0) \in \mathscr{V}
$$

Now let us apply our results to real Hamilton equations (1.4). Let $B_{r}$ be a nonnegative invertible selfadjoint operator on a real Hilbert space $\mathscr{X}_{r}$. Then condition (1.6) holds for $B_{r}$.

Let us consider complexifications $B$ and $J$ of the operators $B_{r}$ and $J_{r}$ in the space $\mathscr{X}=\mathscr{X}_{r}+i \mathscr{X}_{r}$; these are defined by

$$
B\left(X_{1}+i X_{2}\right)=B_{r} X_{1}+i B_{r} X_{2}, \quad J\left(X_{1}+i X_{2}\right)=J_{r} X_{1}+i J_{r} X_{2}, \quad X_{1}, X_{2} \in \mathscr{X}_{r} .
$$

We claim that $B$ satisfies condition (1.6). First, $B$ is invertible since the complexification of $B_{r}^{-1}$ gives $B^{-1}$. Moreover, $B^{-1}$ is a bounded injective selfadjoint operator, and hence $B$ is a densely defined selfadjoint operator in $\mathscr{X}$ by Theorem 13.11 (b) of [47]. Finally, $B$ is obviously nonnegative.

Further, let $\mathscr{V}_{r}$ denote the domain of the real selfadjoint operator $\Lambda_{r}:=B_{r}^{1 / 2}$. Therefore, $\mathscr{V}:=$ $D(\Lambda)=\mathscr{V}_{r}+i \mathscr{V}_{r}$, since $\Lambda:=B^{1 / 2}$ is the complexification of $\Lambda_{r}:=B_{r}^{1 / 2}$. Hence, Corollary 2.3 implies that equation (1.2) admits a unique solution

$$
X(t)=Y_{1}(t)+i Y_{2}(t) \in C(\mathbb{R}, \mathscr{V}), \quad Y_{1}(t), Y_{2}(t) \in \mathscr{V}_{r}
$$

for any $X(0)=X_{r} \in \mathscr{V}_{r}$. The solution admits a spectral representation of type (2.22), where $E(\omega)$ is the spectral family of the corresponding selfadjoint operator $H$ defined by (2.13). Summarizing, Corollary 2.3 and Proposition 2.4 imply the following corollary. Let us denote by $E_{r r}(\omega)$ and $E_{i r}(\omega)$ the real and imaginary components of $\left.E(\omega)\right|_{\mathscr{X}_{r}}$.

Corollary 2.5. Let $B_{r}$ be a nonnegative invertible selfadjoint operator in a real Hilbert space $\mathscr{X}_{r}$. Then

i) A solution $X_{r}(t) \in C\left(\mathbb{R}, \mathscr{V}_{r}\right)$ to (1.4) exists and is unique for any initial state $X_{r}(0) \in \mathscr{V}_{r}$, and the energy (1.5) is conserved.

ii) This solution admits the spectral representation

$$
X_{r}(t)=\Lambda_{r}^{-1} \int\left[\cos \omega t d E_{r r}(\omega)+\sin \omega t d E_{i r}(\omega)\right] \Lambda_{r} X_{r}(0), X_{r}(0) \in \mathscr{V}_{r}
$$

where the Riemann-Stieltjes integral converges in $\mathscr{X}_{r}$. 
Proof $i$ ) First, the real part $Y_{1}(t) \in C\left(\mathbb{R}, \mathscr{V}_{r}\right)$ of solution (2.24) is the unique solution to (1.4) with $Y_{1}(0)=X_{r}$, since the real subspace $\mathscr{X}_{r}$ is invariant with respect to $A_{r}=J_{r} B_{r}$. Similarly, the imaginary part $Y_{2}(t) \in C\left(\mathbb{R}, \mathscr{V}_{r}\right)$ vanishes, since $Y_{2}(0)=0$, and hence the energy conservation for the real solution follows from (2.20) and (1.5).

ii) Formula (2.25) follows from (2.22). The convergence of the Riemann-Stieltjes integral of (2.25) in $\mathscr{X}_{r}$ follows from the convergence in $\mathscr{X}$ of the corresponding integral of (2.22) since the convergence of a sequence in the Hilbert space $\mathscr{X}=\mathscr{X}_{r}+i \mathscr{X}_{r}$ is equivalent to the convergence of the corresponding real and imaginary parts in $\mathscr{X}_{r}$.

\section{Application to eigenfunction expansion}

We are going to apply our results to justification of the eigenfunction expansion (1.7) in the context of the system considered in [30]. We have used this expansion for the calculation of the Fermi Golden Rule [30, (5.14)].

\subsection{Linearization at the kink}

In [30, 31] we studied the 1D relativistic Ginzburg-Landau equation

$$
\ddot{\psi}(x, t)=\frac{d^{2}}{d x^{2}} \psi(x, t)+F(\psi(x, t)), \quad x \in \mathbb{R}
$$

for real solutions $\psi(x, t)$. Here $F(\psi)=-U^{\prime}(\psi)$, where $U(\psi)$ is similar to the Ginzburg-Landau potential $U_{G L}(\psi)=\left(\psi^{2}-1\right)^{2} / 4$, which corresponds to the cubic equation with $F(\psi)=\psi-\psi^{3}$. Namely, $U(\psi)$ is a real smooth even function satisfying the following conditions:

$$
U(\psi)>0, \quad \psi \neq \pm a ; \quad U(\psi)=\frac{m^{2}}{2}(\psi \mp a)^{2}+O\left(|\psi \mp a|^{14}\right), \quad x \rightarrow \pm a,
$$

where $a, m>0$. The main goal of [30, 31] was to prove of the asymptotic stability of solitons (kinks) $\psi(x, t)=s_{v}(x-v t)$ that move with constant velocity $|v|<1$, and

$$
s_{v}(x) \rightarrow \pm a, \quad x \rightarrow \pm \infty
$$

Substituting $\psi(x, t)=s_{v}(x-v t)$ into (3.1), we obtain

$$
v^{2} s_{v}^{\prime \prime}(x)=s_{v}^{\prime \prime}(x)+F\left(s_{v}(x)\right), \quad x \in \mathbb{R} .
$$

The linearization of (3.1) at the kink $s_{v}(x-v t)$ in the moving frame reads as (1.2) with $X=(\psi, \dot{\psi}) \in$ $L^{2}(\mathbb{R}) \otimes \mathbb{C}^{2}$ (for the corresponding complexification) and with the generator [31, (4.6)]

$$
A_{v}=\left(\begin{array}{cc}
v \frac{d}{d x} & 1 \\
\frac{d^{2}}{d x^{2}}-m^{2}-V_{v}(x) & v \frac{d}{d x}
\end{array}\right) .
$$

Here the potential

$$
V_{v}(x)=-F^{\prime}\left(s_{v}(x)\right)-m^{2} \in C^{\infty}(\mathbb{R})
$$


The kink $s_{v}(x)$ is an odd monotonic function in a suitable coordinate $x$, while $F^{\prime}(\psi)=-U^{\prime \prime}(\psi)$ is an even function of $\psi$. Hence, the potential $V_{v}(x)$ is the even function of $x$. Moreover,

$$
\left|V_{v}(x)\right| \leq C e^{-\kappa|x|}, \quad x \in \mathbb{R},
$$

where $\kappa>0$. The generator (3.5) has the form $A_{v}=J B_{v}$ with

$$
B_{v}=\left(\begin{array}{cc}
S_{v} & -v \frac{d}{d x} \\
v \frac{d}{d x} & 1
\end{array}\right), \quad S_{v}:=-\frac{d^{2}}{d x^{2}}+m^{2}+V_{v}(x), \quad J:=\left(\begin{array}{cc}
0 & 1 \\
-1 & 0
\end{array}\right) .
$$

Obviously, $J B_{v} \neq B_{v} J$. Differentiating (3.4), we obtain

$$
\tilde{S}_{v} s_{v}^{\prime}(x)=0, \quad \tilde{S}_{v}:=-\left(1-v^{2}\right) \frac{d^{2}}{d x^{2}}+m^{2}+V_{v}(x)
$$

\subsection{Spectral condition}

Here we check condition (1.6) for operator $B_{v}$ in the space of the odd states in the case $v=0$. The general case $|v|<1$ is considered in Appendix A (see Corollary A.3). We will write $A, B$ and $S$ instead of $A_{0}, B_{0}$ and $S_{0}$ :

$$
A=\left(\begin{array}{cc}
0 & 1 \\
-S & 0
\end{array}\right), \quad B=\left(\begin{array}{cc}
S & 0 \\
0 & 1
\end{array}\right), \quad S:=-\frac{d^{2}}{d x^{2}}+m^{2}+V_{0}(x) .
$$

The operators $B$ and $S$ are essentially selfadjoint in $L^{2}(\mathbb{R}) \otimes \mathbb{C}^{2}$ and $L^{2}(\mathbb{R})$ respectively, by (3.7) and Theorems X.7 and X.8 of [44]. Now (3.9) with $v=0$ means that $\lambda=0 \in \sigma_{p p}(S)$. Moreover, $\lambda=0$ is the minimal eigenvalue of $S$, since the corresponding eigenfunction $s_{0}^{\prime}(x)$ does not vanish [31, (1.9)]. Hence,

$$
\sigma(S) \subset[0, \infty)
$$

Moreover,

$$
\operatorname{Ker} S=\left(s_{0}^{\prime}(x)\right),
$$

since any second linearly independent solution of the homogeneous equation cannot belong to $L^{2}(\mathbb{R})$ by Theorem X.8 of [44].

Below we restrict the phase space $L^{2}(\mathbb{R}) \otimes \mathbb{C}^{2}$ to the invariant subspace of the odd states

$$
\mathscr{X}=\left\{\psi \in L^{2}(\mathbb{R}) \otimes \mathbb{C}^{2}: \psi(-x)=-\psi(x), \quad x \in \mathbb{R}\right\},
$$

as in [30]. The subspace is invariant for the operators $A$ and $B$, since the potential $V_{0}(x)$ is an even function. Respectively, we consider the operator $S$ on the Hilbert space of odd functions $L_{\text {odd }}^{2}(\mathbb{R})$.

Lemma 3.1. Condition (1.6) holds for the operator $B$ on $\mathscr{X}$.

Proof The ground state $s_{0}^{\prime}(x)$ of $S$ on $L^{2}(\mathbb{R}) \otimes \mathbb{C}^{2}$ is an even function. Hence, $\operatorname{Ker} S=0$ for $S$ on $\mathscr{X}$ by (3.12). Further, the continuous spectrum of $S$ lies in $\left[m^{2}, \infty\right)$. Hence, (3.11) implies that

$$
\sigma(S)=\left\{\lambda_{1}, \ldots, \lambda_{N}\right\} \cup\left[m^{2}, \infty\right),
$$

where $0<\lambda_{1}<\ldots<\lambda_{N}<m^{2}$. Finally, $\sigma(B)=\sigma(S) \cup\{1\}$, which implies (1.6).

We will assume below the following spectral condition on the edge point of the continuous spectrum of $S$ imposed in [30]:

The point $m^{2}$ is neither an eigenvalue nor a resonance of $S$. 


\section{Orthogonal eigenfunction expansion}

Let us apply Proposition 2.4 to the case of operators (3.10). We have

$$
\Lambda:=B^{1 / 2}=\left(\begin{array}{cc}
\sqrt{S} & 0 \\
0 & 1
\end{array}\right), \quad H:=\Lambda i J \Lambda=i\left(\begin{array}{cc}
0 & \sqrt{S} \\
-\sqrt{S} & 0
\end{array}\right)=i J \sqrt{S} .
$$

Thus, $H$ is obviously selfadjoint in accordance to Lemma 2.1. Hence, (3.14) implies that

$$
\sigma(H)=(-\infty,-m] \cup\left\{\omega_{-N}, \ldots, \omega_{-1}, \omega_{1}, \ldots, \omega_{N}\right\} \cup[m, \infty), \quad \omega_{ \pm k}^{2}=\lambda_{k}, \quad k=1, \ldots, N
$$

Respectively, formula (2.22) for solutions to (1.2) reads

$$
X(t)=\Lambda^{-1} \int_{\sigma(H)} e^{-i \omega t} d E(\omega) \Lambda X(0)=\sum_{-N}^{N} e^{-i \omega_{k} t} C_{k} a_{k}+\Lambda^{-1} \int_{\sigma_{c}(H)} e^{-i \omega t} d E(\omega) \Lambda X(0)
$$

where $\sigma_{c}(H)=\sigma_{c}=(-\infty,-m] \cup[m, \infty)$ is the continuous spectrum of $H$, and $a_{k}=\Lambda^{-1} h_{k} \in \mathscr{X}$, where $h_{k}$ are the eigenfunctions of $H$ corresponding to the eigenvalues $\omega_{k}$. Formula (2.15) implies that $a_{k}$ are the eigenfunctions of $A$ corresponding to the eigenvalues $-i \omega_{k}$.

Let us denote by $X^{c}(t)$ the last integral in (4.3):

$$
X^{c}(t)=\Lambda^{-1} \int_{\sigma_{c}} e^{-i \omega t} d E(\omega) \Lambda X(0)
$$

To prove (1.7) it remains to justify the eigenfunction expansion

$$
X^{c}(t)=\int_{\sigma_{c}} e^{-i \omega t} C(\omega) a_{\omega} d \omega
$$

where $a_{\omega}$ are the generalized odd eigenfunctions from the continuous spectral space of $A$ corresponding to the eigenvalues $-i \omega$. Then (1.7) will follow from (4.3).

By (4.3), $X^{c}(t)$ is the solution to (1.2), and hence $Z^{c}(t):=\Lambda X^{c}(t)$ is the solution to (2.10). We will deduce (4.5) from the corresponding representation

$$
Z^{c}(t)=\int_{\sigma_{c}} e^{-i \omega t} C(\omega) h_{\omega} d \omega
$$

where $h_{\omega}$ are the generalized odd eigenfunctions of $H$ with the eigenvalues $\omega$. We will prove (4.6) by solving equation (2.10) for $Z^{c}(t)=\left(Z_{1}^{c}(t), Z_{2}^{c}(t)\right)$. By (4.1), this equation is equivalent to the system

$$
\dot{Z}_{1}^{c}(t)=\sqrt{S} Z_{2}^{c}(t), \quad \dot{Z}_{2}^{c}(t)=-\sqrt{S} Z_{1}^{c}(t) .
$$

Eliminating $Z_{2}^{c}$, we obtain

$$
\ddot{Z}_{1}^{c}(t)=-S Z_{1}^{c}(t)
$$

Further we apply Theorem XI.41 of [45] and the arguments of [45, pp 114-115]. Namely, the rapid decay (3.7) and our spectral condition (3.15) imply the following Limiting Absorption Principle (LAP) [1, 32, 45]:

$$
R(\lambda \pm i \varepsilon) \rightarrow R_{ \pm}(\lambda), \quad \varepsilon \rightarrow+0, \quad \lambda \in\left[m^{2}, \infty\right)
$$


where $R(z):=(S-z)^{-1}$ and the convergence holds in the strong topology of the space of continuous operators $L_{s}^{2} \rightarrow L_{-s}^{2}$ with $s>1$. Moreover, the traces of the resolvent $R_{ \pm}(\lambda)$ are continuous functions of $\lambda \geq m^{2}$ with values in $L\left(L_{s}^{2}, L_{-s}^{2}\right)$, see [1, 32]. Here $L_{\rho}^{2}=L_{\rho}^{2}(\mathbb{R})$ with $\rho \in \mathbb{R}$ denotes the weighted Hilbert space with the norm

$$
\|\psi\|_{L_{\rho}^{2}}^{2}:=\int\langle x\rangle^{2 \rho}|\psi(x)|^{2} d x, \quad\langle x\rangle:=\left(1+x^{2}\right)^{1 / 2} .
$$

The LAP serves as the basis for the eigenfunction expansion

$$
Z_{1}^{c}(t)=\int_{\sigma_{c}} d \mathscr{E}\left(\omega^{2}\right)\left[Z_{1}^{c}(0) \cos \omega t+Z_{2}^{c}(0) \sin \omega t\right]=\int_{\sigma_{c}} e^{-i \omega t} C(\omega) e_{\omega} d \omega
$$

where $d \mathscr{E}(\lambda)$ is the spectral resolution of $S$ in the space $L_{\text {odd }}^{2}(\mathbb{R})$, while $e_{\omega} \in L_{-S}^{2}$ are generalized odd eigenfunctions of $S$ corresponding to the eigenvalues $\omega^{2} \geq m^{2}$. Here the first identity follows by Spectral Theorem, while the second follows by Theorem XI.41 (e) of [45]. The eigenfunctions are defined by formulas of [45, pp 114-115]:

$$
e_{\omega}=W^{*}(\omega) f_{\omega}, f_{\omega}(x):=\sin |\omega| x, W(\omega):=\left[1+V R_{0}\left(\omega^{2}+i 0\right)\right]^{-1}, \omega \in \sigma_{c} .
$$

where $R_{0}(\lambda):=\left(-\Delta+m^{2}-\lambda\right)^{-1}$. The operator $W(\omega)$ is a continuous function of $\omega \in \sigma_{c}$ with values in $L\left(L_{s}^{2}, L_{s}^{2}\right)$ by the formula

$$
\left[1+V R_{0}(\lambda)\right]^{-1}=1-V R(\lambda)
$$

and the decay (3.7). Respectively, the adjoint operator $W^{*}(\omega)$ is a continuous function of $\omega \in \sigma_{c}$ with values in $L\left(L_{-s}^{2}, L_{-s}^{2}\right)$. As the result, $e_{\omega}$ is a continuous function of $\omega \in \sigma_{c}$ with values in $L_{-s}^{2}$. The normalization of $e_{\omega}$ coincides with the same of the 'free' generalized eigenfunctions $f_{\omega}$ :

$$
\left\langle e_{\omega}, e_{\omega^{\prime}}\right\rangle=\pi \delta\left(|\omega|-\left|\omega^{\prime}\right|\right), \quad \omega, \omega^{\prime} \in \sigma_{c},
$$

which follows from the last formula on page 115 of [45]. Finally, Theorem XI.41 (e) of [45] implies that the last integral (4.11) converges in $L^{2}=L^{2}(\mathbb{R})$ :

$$
\left\|Z_{1}^{c}(t)-\int_{m \leq|\omega| \leq M} e^{-i \omega t} C(\omega) e_{\omega} d \omega\right\|_{L^{2}} \rightarrow 0, \quad M \rightarrow \infty
$$

Remark 4.1. Our modification of the eigenfunction expansion theory differs from [45, pp 114-115] only in the use of Hilbert spaces with weights $\langle x\rangle^{s}$ instead of $e^{s|x|}$. All conclusions of Theorem XI.41 from [45] remain valid in the modified theory. This modification allows us to apply Lemma 5.2 below.

Now (4.6) for $Z_{1}^{c}(t)$ follows from (4.11). For $Z_{2}^{c}(t)$ we use the first equation of (4.7), which implies

$$
Z_{2}^{c}(t)=-i \int_{\sigma_{c}} \operatorname{sgn} \omega e^{-i \omega t} C(\omega) e_{\omega} d \omega
$$

Combining (4.11) and (4.16), we obtain (4.6) with

$$
h_{\omega}:=\left(\begin{array}{c}
1 \\
-i \operatorname{sgn} \omega
\end{array}\right) e_{\omega} \in L_{-s}^{2} \otimes \mathbb{C}^{2}, \quad \omega \in \sigma_{c}, \quad \forall s>1 .
$$

Normalization (4.14) implies the corresponding normalization for $h_{\omega}$ :

$$
\left\langle h_{\omega}, h_{\omega^{\prime}}\right\rangle=2 \pi \delta\left(\omega-\omega^{\prime}\right), \quad \omega, \omega^{\prime} \in \sigma_{c} .
$$


Lemma 4.2. Let condition (3.15) hold and $s>1$. Then

i) $h_{\omega}$ are generalized eigenfunctions of $H$, i.e.,

$$
H Z^{c}(t)=\int_{\sigma_{c}} e^{-i \omega t} \omega C(\omega) h_{\omega} d \omega \quad \text { for } \quad Z^{c}(t) \in D(H) .
$$

ii) $h_{\omega}$ is a continuous function of $\omega \in \sigma_{c}$ with values in $L_{-s}^{2} \otimes \mathbb{C}^{2}$.

iii) The integral (4.6) converges in $L^{2} \otimes \mathbb{C}^{2}$ in the following sense:

$$
\left\|Z^{c}(t)-\int_{m \leq|\omega| \leq M} e^{-i \omega t} C(\omega) h_{\omega} d \omega\right\|_{L^{2} \otimes \mathbb{C}^{2}} \rightarrow 0, \quad M \rightarrow \infty .
$$

Proof i) $Z^{c}(t) \in D(H)$ means that $Z_{1,2}^{c}(t) \in D(\sqrt{S})$. Furthermore,

$$
H Z^{c}(t)=i \sqrt{S}\left(\begin{array}{c}
Z_{2}^{c}(t) \\
-Z_{1}^{c}(t)
\end{array}\right)
$$

Now (4.19) follows from the expansions (4.11) and (4.16) for $Z_{1,2}^{c}(t)$ by [45, Theorem XI.41 (c)], since $e_{\omega}$ are the generalized eigenfunctions of $S$ with the eigenvalues $\omega^{2}$, and formally,

$$
i \sqrt{S}\left(\begin{array}{c}
-i \operatorname{sgn} \omega \\
-1
\end{array}\right) e_{\omega}=\left(\begin{array}{c}
\operatorname{sgn} \omega \\
-i
\end{array}\right)|\omega| e_{\omega}=\omega h_{\omega} .
$$

ii) $h_{\omega}$ is a continuous function of $\omega \in \sigma_{c}$ by similar property of $e_{\omega}$.

iii) (4.20) follows from 4.15) and similar convergence for $Z_{2}^{c}$.

Remark 4.3. The generalized eigenfunctions (4.17) are proportional to $e_{\omega}$ since $H$ and $S$ commute with each other. This argument was the main idea of our derivation of the eigenfunctions (4.17).

\section{Non-orthogonal eigenfunction expansion}

Let us denote by $Z_{M}^{c}(t, x)$ the integral in (4.20). It is defined for almost all $x$, i.e.,

$$
Z_{M}^{c}(t, x):=\int_{m \leq|\omega| \leq M} e^{-i \omega t} C(\omega) h_{\omega}(x) d \omega, \quad \text { a.a. } x \in \mathbb{R} .
$$

To justify (4.5) we should adjust the meaning of this integral by the following lemma.

Lemma 5.1. Let condition (3.15) hold and $s>1$. Then

i) The integral (5.1) converges absolutely in $L_{-s}^{2} \otimes \mathbb{C}^{2}$ :

$$
\int_{m \leq|\omega| \leq M}\left\|C(\omega) h_{\omega}\right\|_{L_{-s}^{2} \otimes \mathbb{C}^{2}} d \omega<\infty, \quad M>m
$$

ii) The integral (5.1) coincides a.e. with the corresponding integral of the $L_{-s}^{2} \otimes \mathbb{C}^{2}$-valued integrand. 
Proof i) (4.18) and the Plancherel identity [45, $\left.\left(82 \mathrm{e}^{\prime}\right)\right]$ imply that

$$
\left\|\int_{\alpha}^{\beta} C(\omega) h_{\omega} d \omega\right\|_{\mathscr{X}}^{2}=2 \pi \int_{\alpha}^{\beta}|C(\omega)|^{2} d \omega=\left\|P_{[\alpha, \beta]} Z^{c}(0)\right\|_{\mathscr{X}}^{2} \leq\|Z(0)\|_{\mathscr{X}}^{2}, \quad[\alpha, \beta] \subset \sigma_{c} .
$$

Hence, (5.2) follows by the Cauchy-Schwarz inequality and Lemma 4.2 i).

ii) The scalar products of the both integrals with any test function $\varphi \in C_{0}^{\infty}(\mathbb{R})$ coincide by the Fubini theorem since $h_{\omega}(x)$ can be chosen a measurable function of $(\omega, x) \in \sigma_{c} \times \mathbb{R}$ by Lemma $\left.4.2 \mathrm{ii}\right)$.

Now we are going to deduce (4.5) applying $\Lambda^{-1}$ to (4.6).

Lemma 5.2. The operator $\Lambda^{-1}: L_{\rho}^{2} \otimes \mathbb{C}^{2} \rightarrow L_{\rho}^{2} \otimes \mathbb{C}^{2}$ is continuous for every $\rho \in \mathbb{R}$.

Proof By (4.1) we have

$$
\Lambda^{-1}=\left(\begin{array}{cc}
S^{-1 / 2} & 0 \\
0 & 1
\end{array}\right)
$$

Hence, it suffices to prove the continuity for the operator $S^{-1 / 2}$ in $L_{\rho}^{2}$, which means the continuity of operator

$$
\langle x\rangle^{\rho} S^{-1 / 2}\langle x\rangle^{-\rho}: L^{2}(\mathbb{R}) \rightarrow L^{2}(\mathbb{R}) .
$$

This continuity follows by the Theorem of Composition of PDO, since $S^{-1 / 2}$ is a PDO of the class $H G_{1}^{-1,-1}$, see definition 25.2 in [50]. This follows from [20, Theorem 29.1.9] and also by an extension of [50, Theorem 11.2] to PDO with nonempty continuous spectrum. It is important that operator $S$ and its main symbol $\xi^{2}$ satisfy

$$
\xi^{2} \notin(-\infty, 0], \quad \xi \neq 0 ; \quad \sigma(S) \cap(-\infty, 0]=\emptyset .
$$

Hence, conditions (10.1) and (10.2) of [50] hold.

Lemma 5.2 and (4.17) imply that

$$
a_{\omega}:=\Lambda^{-1} h_{\omega} \in L_{-s}^{2} \otimes \mathbb{C}^{2}, \quad s>1 .
$$

Lemma 5.3. $a_{\omega}$ are generalized eigenfunctions of $A$ corresponding to the eigenvalues $-i \omega$.

Proof Let $P_{c}^{M}$ denote the spectral projection $\chi_{c}^{M}(H)$, where $\chi_{c}^{M}$ is the indicator of $\sigma_{c}^{M}:=\sigma_{c} \cap$ $[-M, M]$. Let us take any $Z^{c}(0) \in P_{c}^{M} \mathscr{X}$. Then $Z^{c}(0) \in D(H)$, and hence (4.6) and (4.19) with $t=0$ imply the expansions

$$
Z^{c}(0)=\int_{\sigma_{c}^{M}} C(\omega) h_{\omega} d \omega, \quad H Z^{c}(0)=\int_{\sigma_{c}^{M}} \omega C(\omega) h_{\omega} d \omega .
$$

Now let us take any $X \in \Lambda^{-1} P_{c}^{M} \mathscr{X}$ and write (5.6) for $Z^{c}(0)=\Lambda X$. Applying $\Lambda^{-1}$ to each side, we obtain

$$
X=\int_{\sigma_{c}^{M}} C(\omega) a_{\omega} d \omega, \quad \Lambda^{-1} H \Lambda X=A X=-i \int_{\sigma_{c}^{M}} \omega C(\omega) a_{\omega} d \omega,
$$

where we have used definition (5.5), Lemmas 5.1 and 5.2, and the expression (2.15) for $A$. Identities (5.7) mean that $a_{\omega}$ are generalized eigenfunctions in the sense of [45, (80b)].

Finally, the main result of our paper is the following. 
Theorem 5.4. Let condition (3.15) hold, $X(0) \in \mathscr{V}$ and $s>1$. Then eigenfunction expansion (4.5) holds in the following sense:

$$
\left\|X^{c}(t)-\int_{m \leq|\omega| \leq M} e^{-i \omega t} C(\omega) a_{\omega} d \omega\right\|_{\mathscr{V}} \rightarrow 0, \quad M \rightarrow \infty
$$

where the integral converges in $L_{-s}^{2} \otimes \mathbb{C}^{2}$, and hence, a.e., as in (5.1).

Proof We should prove that

$$
\left\|\Lambda X^{c}(t)-\Lambda \int_{m \leq|\omega| \leq M} e^{-i \omega t} C(\omega) a_{\omega} d \omega\right\|_{\mathscr{X}} \rightarrow 0, \quad M \rightarrow \infty .
$$

First, we recall that $\Lambda X^{c}(t)=Z^{c}(t)$. Second,

$$
\int_{m \leq|\omega| \leq M} e^{-i \omega t} C(\omega) a_{\omega} d \omega=\Lambda^{-1} \int_{m \leq|\omega| \leq M} e^{-i \omega t} C(\omega) h_{\omega} d \omega
$$

by definition (5.5) and Lemmas 5.1, 5.2. Now (5.9) follows from (4.20).

\section{Symplectic normalization}

Now let us renormalize $h_{\omega}$ as follows:

$$
\left\langle h_{\omega}, h_{\omega^{\prime}}\right\rangle=|\omega| \delta\left(\omega-\omega^{\prime}\right), \quad \omega, \omega^{\prime} \in \sigma_{c} .
$$

This means that for any $M<\infty$

$$
\left\langle Z_{1}, Z_{2}\right\rangle=\int_{m \leq|\omega| \leq M}|\omega| C_{1}(\omega) \overline{C_{2}(\omega)} d \omega, \quad \text { for } \quad Z_{1,2}=\int_{m \leq|\omega| \leq M} C_{1,2}(\omega) h_{\omega} d \omega \in \mathscr{X} .
$$

Let us express these formulas in terms of $X_{1,2}:=\Lambda^{-1} Z_{1,2} \in \mathscr{V}$ and the eigenfunctions $a_{\omega}:=\Lambda^{-1} h_{\omega}$.

First, 4.19) and (6.2) imply that

$$
\left\langle H^{-1} Z_{1}, Z_{2}\right\rangle=\int_{m \leq|\omega| \leq M} \operatorname{sgn} \omega C_{1}(\omega) \overline{C_{2}(\omega)} d \omega .
$$

On the other hand, (2.13) implies that

$$
\left\langle H^{-1} Z_{1}, Z_{2}\right\rangle=\left\langle\Lambda^{-1} i J \Lambda^{-1} Z_{1}, Z_{2}\right\rangle=-i\left\langle\Lambda^{-1} Z_{1}, J \Lambda^{-1} Z_{2}\right\rangle=-i\left\langle X_{1}, J X_{2}\right\rangle .
$$

Finally, 6.3) - 6.4) imply that

$$
-i\left\langle X_{1}, J X_{2}\right\rangle=\int_{m \leq|\omega| \leq M} \operatorname{sgn} \omega C_{1}(\omega) \overline{C_{2}(\omega)} d \omega \quad \text { for } \quad X_{1,2}=\int_{m \leq|\omega| \leq M} C_{1,2}(\omega) a_{\omega} d \omega .
$$

By definition, this means that

$$
\left\langle a_{\omega}, J a_{\omega^{\prime}}\right\rangle=i \operatorname{sgn} \omega \delta\left(\omega-\omega^{\prime}\right), \quad \omega, \omega^{\prime} \in \sigma_{c} .
$$

Now the expansion (4.5) coincides with [7, (2.1.13)], thereby justifying our calculation of the Fermi Golden Rule [30, (5.14)]. 


\section{A Spectral condition for $|v|<1$}

Let us check the spectral condition (1.6) for operators $B_{v}$ from (3.8) with any $|v|<1$ in the space of the odd states. First let us check the continuous spectrum.

Lemma A.1. The continuous spectrum of $B_{v}$ lies in $[\delta, \infty)$ with some $\delta>0$.

Proof By Corollary 2 (c) of [46, XIII.4] and (3.7) it suffices to find the continuous spectrum of the unperturbed operator $B_{v}^{0}$ corresponding to $V_{v}(x)=0$. Consider the spectral equation

$$
\left(B_{v}^{0}-\lambda\right) \psi=0
$$

and find the solution of type $\psi=e^{i k x} \phi$ with real $k$ and $\phi \in \mathbb{C}^{2}$. Substituting to A. 1) we obtain

$$
\left(\begin{array}{cc}
k^{2}+m^{2}-\lambda & i k v \\
-i k v & 1-\lambda
\end{array}\right) \phi=0 .
$$

For nonzero vectors $\phi$, the determinant of the matrix vanishes:

$$
k^{2}\left(1-\lambda-v^{2}\right)+\left(m^{2}-\lambda\right)(1-\lambda)=0 .
$$

Then $k^{2}=\left(m^{2}-\lambda\right)(1-\lambda) /\left(\lambda-1+v^{2}\right) \geq 0$. This inequality holds if

$$
\left\{\begin{array}{lll}
\lambda \in\left[1-v^{2}, 1\right] \cup\left[m^{2}, \infty\right) & \text { for } & 1 \leq m^{2}, \\
\lambda \in\left[m^{2}, 1-v^{2}\right) \cup(1, \infty) & \text { for } & m^{2} \leq 1-v^{2} \leq 1, \\
\lambda \in\left[1-v^{2}, m^{2}\right] \cup[1, \infty) & \text { for } & 1-v^{2} \leq m^{2} \leq 1 .
\end{array}\right.
$$

Now the lemma follows with $\delta=\min \left(1-v^{2}, m^{2}\right)$.

Now let us consider the discrete spectrum.

Lemma A.2. $i) \operatorname{dim} \operatorname{Ker} B$ is generated by $\left(s_{0}^{\prime}(x),-v s_{0}^{\prime \prime}(x)\right)$, where $s_{0}^{\prime}(x)$ is an even function.

ii) The nonzero discrete spectrum of $B$ is positive.

Proof $i$ ) Equation $B \psi=0$ is equivalent to the system

$$
\left(\begin{array}{cc}
S_{v} & -v \frac{d}{d x} \\
v \frac{d}{d x} & 1
\end{array}\right)\left(\begin{array}{l}
\psi_{1} \\
\psi_{2}
\end{array}\right)=0 .
$$

The second equation (A. 7) implies $\psi_{2}=-v \psi_{1}^{\prime}$. Substituting into the first equation we obtain

$$
\tilde{S}_{v} \psi_{1}=0
$$

where $\tilde{S}_{v}$ is defined in (3.9). However, the equation (3.9) with $v=0$ means that $\lambda=0 \in \sigma_{p p}\left(\tilde{S}_{v}\right)$. Moreover, $\lambda=0$ is the minimal eigenvalue of $\tilde{S}_{v}$, since the corresponding eigenfunction $s_{v}^{\prime}(x)$ does not vanish [31, (1.9)]. Hence,

$$
\sigma\left(\tilde{S}_{v}\right) \subset[0, \infty)
$$


Moreover,

$$
\operatorname{Ker} \tilde{S}_{v}=\left(s_{0}^{\prime}(x)\right)
$$

since any second linearly independent solution of the homogeneous equation cannot belong to $L^{2}(\mathbb{R})$ by Theorem X.8 of [44].

Step ii) Consider equation $B_{v} \psi=\lambda \psi$ with $\lambda<0$ :

$$
\left(\begin{array}{cc}
S_{v}-\lambda & -v \frac{d}{d x} \\
v \frac{d}{d x} & 1-\lambda
\end{array}\right)\left(\begin{array}{l}
\psi_{1} \\
\psi_{2}
\end{array}\right)=0 .
$$

The second equation (A. 7) implies $\psi_{2}=v \psi_{1}^{\prime} /(\lambda-1)$. Substituting into the first equation we obtain

$$
\left(\tilde{S}_{v}+\frac{v^{2} \lambda}{1-\lambda} \frac{d^{2}}{d x^{2}}-\lambda\right) \psi_{1}=0 .
$$

For $\lambda<0$ the operator is positive since $\tilde{S}_{v} \geq 0$ by (A. 5). Hence, equation (A. 8) has no nonzero solutions $\psi_{1} \in L^{2}$.

Corollary A.3. Lemmas 3.1 and A.2 imply that condition (1.6) holds for $|v|<1$ in the space of the odd states.

\section{B Linearization of $U(1)$-invariant Hamilton PDEs}

Equations (1.2) with $J B \neq B J$ arise in the linearization of nonlinear $U(1)$-invariant Hamilton PDEs. Namely, consider the $U(1)$-invariant Hamilton functional

$$
\mathscr{H}(\psi)=\frac{1}{2} \int\left[|\nabla \psi(x)|^{2}+U\left(x,|\psi(x)|^{2}\right)\right] d x
$$

with a real potential $U(x, r)$ and $\psi(x) \in \mathbb{C}=\mathbb{R}^{2}$. The corresponding Hamilton equation reads as the nonlinear Schrödinger equation

$$
i \dot{\psi}(x, t)=\nabla_{\bar{\psi}} \mathscr{H}(\psi)=-\Delta \psi(x, t)+U_{r}\left(x,|\psi|^{2}\right) \psi, \quad x \in \mathbb{R}^{n},
$$

where $i$ can be regarded as a real $2 \times 2$ matrix $J$ of type (3.8). The linearization at a stationary state $s_{0}(x)$ is obtained by substitution $\psi=s_{0}+\varphi$ and expansion $|\psi|^{2}=\left|s_{0}\right|^{2}+2 s_{0} \cdot \varphi+|\varphi|^{2}$, where $s_{0} \cdot \varphi$ is the scalar product of the real vectors from $\mathbb{R}^{2}$. Neglecting the terms of higher order, we obtain the linearized equation

$$
i \dot{\varphi}(x, t)=-\Delta \varphi(x, t)+U_{r}\left(x,\left|s_{0}(x)\right|^{2}\right) \varphi+2 U_{r r}\left(x,\left|s_{0}(x)\right|^{2}\right)\left(s_{0} \cdot \varphi\right) s_{0}
$$

which can be represented in the form (1.2) with $J=-i$ and $X(t)=(\operatorname{Re} \varphi(t), \operatorname{Im} \varphi(t))$. The last term of $(\mathrm{B}$. 11) is not complex linear operator of $\varphi$. In other words, it does not commute with the multiplication of $\varphi$ by $i$. So $J B \neq B J$ if $U_{r r}\left(x,\left|s_{0}(x)\right|^{2}\right) \not \equiv 0$. Let us assume that $U(x, r)$ is a realanalytic function of $r>0$, and $s_{0}(x) \not \equiv 0$. Then the last term of (B. 11) vanishes exactly for the linear Schrödinger equation when $U(x, r)=V(x) r$. 


\section{References}

[1] S. Agmon, Spectral properties of Schrödinger operator and scattering theory, Ann. Scuola Norm. Sup. Pisa, Ser. IV 2, 151-218 (1975).

[2] T.Ya. Azizov, I.S. Iokhvidov, Linear Operators in Space with an Indefinite Metric, John Wiley \& Sons, Chichester, 1989.

[3] D. Bambusi, S. Cuccagna, On dispersion of small energy solutions of the nonlinear KleinGordon equation with a potential, Amer. J. Math. 133 (2011), no. 5, 1421-1468.

[4] N. Boussaid, S. Cuccagna, On stability of standing waves of nonlinear Dirac equations, Comm. PDE 37 (2012), no. 6, 1001-1056. arXiv:1103.4452.

[5] V.S. Buslaev, G.S. Perelman, Scattering for the nonlinear Schrödinger equation: states close to a soliton, St.Petersburg Math. J. 4 (1993), 1111-1142.

[6] V.S. Buslaev, G.S. Perelman, On the stability of solitary waves for nonlinear Schrödinger equations, Nonlinear evolution equations, Transl. Ser. 2, 164, Amer. Math. Soc., Providence, RI, 1995, pp. 75-98.

[7] V.S. Buslaev, C. Sulem, On asymptotic stability of solitary waves for nonlinear Schrödinger equations, Ann. Inst. Henri Poincaré, Anal. Non Linéaire 20 (2003), no. 3, 419-475.

[8] T. Cazenave, A. Haraux, Semilinear evolution equations, Clarendon Press, Oxford, 1998.

[9] S. Cuccagna, Stabilization of solutions to nonlinear Schrödinger equations, Comm. Pure Appl. Math. 54 (2001), 1110-1145.

[10] S. Cuccagna, On asymptotic stability of ground states of NLS, Rev. Math. Phys. 15 (2003), 877-903.

[11] S. Cuccagna, D. Pelinovsky,V. Vougalter, Spectra of positive and negative energies in the linearized NLS problem, Commun. Pure Appl. Math. 58 (2005), no. 1, 1-29.

[12] S. Cuccagna, On scattering of small energy solutions of non-autonomous Hamiltonian nonlinear Schrödinger equations, J. Differ. Equations 250 (2011), no. 5, 2347-2371.

[13] S. Cuccagna, The Hamiltonian structure of the nonlinear Schrödinger equation and the asymptotic stability of its ground states, Commun. Math. Phys. 305 (2011), no. 2, 279-331.

[14] S. Cuccagna, On asymptotic stability of moving ground states of the nonlinear Schrödinger equation, To appear in Trans. Amer. Math. Soc, 2012. arXiv:1107.4954

[15] S. Cuccagna, T. Mizumachi, On asymptotic stability in energy space of ground states for nonlinear Schrödinger equations, Commun. Math. Phys. 284 (2008), no. 1, 51-77.

[16] S. Cuccagna, M. Tarulli, On asymptotic stability in energy space of ground states of NLS in 2D, Ann. Inst. Henri Poincaré, Anal. Non Linéaire 26 (2009), no. 4, 1361-1386. 
[17] M.B. Erdogan, W. Schlag, Dispersive estimates for Schrdinger operators in the presence of a resonance and/or an eigenvalue at zero energy in dimension three. II, J. Anal. Math. 99(2006), 199-248.

[18] C. Gérard, Scattering theory for Klein-Gordon equations with non-positive energy, Ann. Henri Poincaré 13 (2012), no. 4, 883-941.

[19] I.C. Gohberg, M.G. Krein, Theory and applications of Volterra operators in Hilbert space, AMS, Providence, R.I., 1970.

[20] L. Hörmander, The analysis of linear partial differential operators. IV: Fourier integral operators, Springer, Berlin, 2009.

[21] I.S. Iohvidov, M.G. Krein, H. Langer, Introduction to the spectral theory of operators in spaces with an indefinite metric, Mathematical Research, Vol. 9, Akademie-Verlag, Berlin, 1982.

[22] P. Jonas, On the functional calculus and the spectral function for definitizable operators in Krein space Beitr. Anal. 16(1981), 121-135.

[23] P. Jonas, On a class of unitary operators in Krein space, pp 151-172 in: Operator Theory: Advances and Applications, Vol.17, Birkhäuser Verlag, Basel-Boston-Stuttgart, 1986.

[24] P. Jonas, On a class of selfadjoint operators in Krein space and their compact perturbations, Integral Equ. Oper. Theory 11 (1988), 351-384.

[25] P. Jonas, On the spectral theory of operators associated with perturbed Klein- Gordon and wave type equations, J. Oper. Theory 29 (1993), 207-224.

[26] P. Jonas, On bounded perturbations of operators of Klein-Gordon type, Glasnik Math. 35 (2000), 59-74.

[27] Kako, T.: Spectral and scattering theory for the J-selfadjoint operators associated with the perturbed Klein- Gordon type equations. J. Fac. Sci. Univ. Tokyo Sec. I A 23, 199221 (1976)

[28] V. Imaykin, A.I. Komech, H. Spohn, Scattering asymptotics for a charged particle coupled to the Maxwell field, J. Math. Physics 52 (2011), no. 4, 042701-042701-33. arXiv:0807.1972

[29] A.I. Komech, E.A. Kopylova, H. Spohn, Scattering of solitons for Dirac equation coupled to a particle, J. Math. Analysis and Appl. 383 (2011), no. 2, 265-290. arXiv: 1012.3109

[30] E.A. Kopylova, A.I. Komech, On asymptotic stability of kink for relativistic Ginzburg-Landau equation, Arch. Rat. Mech. Anal. 202 (2011), no. 2, 213-245. arXiv:0910.5539

[31] E.A. Kopylova, A.I. Komech, On asymptotic stability of moving kink for relativistic GinzburgLandau equation, Comm. Math. Physics 302 (2011), no.1, 225-252. arXiv:0910.5538

[32] A. Komech, E.A. Kopylova, Dispersion decay and scattering theory, Wiley, Hoboken, NJ, 2012.

[33] M.G. Krein, H.K. Langer, The spectral function of a selfadjoint operator in a space with indefinite metric, Sov. Math. Dokl. 4 (1963), 1236-1239. 
[34] M.G. Krein, Yu. Shmul'jan, J-polar representations of plus-operators, Mat. Issled. 1 (1966), no.2, 172-210. [Russian]

[35] H. Langer, Spectral functions of definitizable operators in Krein spaces, pp. 1-46 in: D. Butkovic, H. Kraljevic, S. Kurepa, Functional Analysis, LNM0948, Berlin, Springer, 1981.

[36] Langer, H.; Najman, B.: Perturbation theory for definitizable operators in Krein spaces, J.Operator Theory 9 (1983), 297-317.

[37] H. Langer, B. Najman, C. Tretter, Spectral theory of the Klein-Gordon equation in Krein spaces, Proc. Edinb. Math. Soc., II. Ser. 51 (2008), no. 3, 711-750.

[38] H. Langer, B. Najman, C. Tretter, Spectral theory of the Klein-Gordon equation in Pontryagin spaces, Commun. Math. Phys. 267 (2006), no. 1, 159-180.

[39] H. Langer, C. Tretter, Variational principles for eigenvalues of the Klein-Gordon equation, $J$. Math. Phys. 47 (2006), no. 10, 103506, 18 p.

[40] Y. Martel, F. Merle, Asymptotic stability of solitons of the gKdV equations with general nonlinearity, Math. Ann. 341 (2008), 391-427.

[41] J. Miller, M. Weinstein, Asymptotic stability of solitary waves for the regularized long-wave equation, Comm. Pure Appl. Math. 49 (1996), 399-441.

[42] R.L. Pego, M.I. Weinstein, Asymptotic stability of solitary waves, Comm. Math. Phys. 164 (1994), 305-349.

[43] M. Reed, B. Simon, Methods of modern mathematical physics I: Functional Analysis, Academic Press, NY, 1980.

[44] M. Reed, B. Simon, Methods of modern mathematical physics II: Fourier Analysis, SelfAdjointness, Academic Press, NY, 1975.

[45] M. Reed, B. Simon, Methods of modern mathematical physics III: Scattering Theory, Academic Press, NY, 1979.

[46] M. Reed, B. Simon, Methods of modern mathematical physics IV: Analysis of Operators, Academic Press, NY, 1978.

[47] W. Rudin, Functional analysis, McGraw-Hill, New York, 1991.

[48] J.J.Sakurai, Advanced Quantum Mechanics, Addison-Wesley, Reading, Mass., 1967.

[49] W.Schlag, Dispersive estimates for Schrdinger operators: a survey, pp 255-285 in: J. Bourgain (ed.) et al., Mathematical aspects of nonlinear dispersive equations. Lectures of the CMI/IAS workshop on mathematical aspects of nonlinear PDEs, Princeton, NJ, USA, 2004. NJ: Princeton University Press, Princeton, 2007.

[50] M.A. Shubin, Pseudodifferential operators and spectral theory, Springer, NY, 1987. 
[51] R.T. Seeley, Complex powers of an elliptic operator, Proc. Sympos. Pure Math. 10 (1967), 288307.

[52] I.M. Sigal, Nonlinear wave and Schrödinger equations. I: Instability of periodic and quasiperiodic solutions, Commun. Math. Phys. 153 (1993), no.2, 297-320.

[53] A. Soffer, M.I. Weinstein, Multichannel nonlinear scattering in nonintegrable systems, Comm. Math. Phys. 133 (1990), 119-146.

[54] A. Soffer, M.I. Weinstein, Multichannel nonlinear scattering and stability II. The case of anisotropic and potential and data, J. Differential Equations 98 (1992), 376-390.

[55] A. Soffer, M.I. Weinstein, Resonances, radiation damping and instability in Hamiltonian nonlinear wave equations, Invent. Math. 136 (1999), 9-74.

[56] A. Soffer, M.I. Weinstein, Selection of the ground state for nonlinear Schrödinger equations, Rev. Math. Phys. 16 (2004), no. 8, 977-1071. arXiv:nlin/0308020.

[57] H. Spohn, Dynamics of charged particles and their radiation field, Cambridge University Press, Cambridge, 2004.

[58] Tai-Peng Tsai, Asymptotic dynamics of nonlinear Schrödinger equations with many bound states, J. Differ. Equations 192 (2003), no. 1, 225-282. 